\title{
Reproduction and Offspring Sex Ratios Differ Markedly among Closely Related Hyperparasitoids Living in the Same Microhabitats
}

\author{
Jeffrey A. Harvey (D) L ucas de Haan • \\ Oriol Verdeny-Vilalta $•$ Bertanne Visser $•$ Rieta Gols
}

Received: 2 May 2019 /Revised: 2 October 2019/Accepted: 12 October 2019 / Published online: 25 October 2019

(C) The Author(s) 2019

\begin{abstract}
Closely related species in nature usually exhibit very similar phylogenetically conserved traits, such as reproduction, behavior and development. Here, we compared fecundity schedules, lifetime reproductive success and offspring sex ratios in three congeneric facultative hyperparasitoid wasps that exhibit several overlapping traits and which co-occur in the same small-scale habitats. Gelis agilis, G. proximus and G. hortensis are abundant in meadows and forest edge habitats in the Netherlands. Gelis agilis is asexual (all female), whereas the other two species reproduce sexually. Here they developed on cocoons of the primary parasitoid Cotesia glomerata. When provided with unlimited hosts, lifetime reproductive success was three
\end{abstract}

\footnotetext{
J. A. Harvey $(\bowtie)$

Department of Ecological Science, Section Animal Ecology, VU University Amsterdam, Amsterdam the Netherlands

e-mail: j.harvey@nioo.knaw.nl

J. A. Harvey · L. de Haan

Department of Terrestrial Ecology, Netherlands Institute of

Ecology, Droevendaalsesteeg 106708 PB Wageningen

The Netherlands

O. Verdeny-Vilalta

Ynsect, Rue Pierre Fontaine 1 Évry France

B. Visser

Evolutionary Ecology and Genetics Group, Biodiversity Research Centre, Earth and Life Institute, UC Louvain, Croix du Sud 4 1348 Louvain-la-Neuve Belgium

R. Gols

Laboratory of Entomology, Wageningen University,

Droevendaalsesteeg 16708 PB Wageningen The Netherlands
}

times higher in $G$. proximus than in G. agilis with $G$. hortensis producing intermediate numbers of offspring. All three species depleted their teneral reserves during their lives. Females of G. proximus and $G$. hortensis lived significantly longer than females of $G$. agilis. Offspring sex ratios in young $G$. proximus mothers were female-biased and marginally malebiased in $G$. hortensis. As mothers aged, however, the ratio of male:female progeny produced rapidly increased until no daughters emerged later in life. Our results reveal significant differences in reproductive traits among the three species despite them cooccurring in the same microhabitats, being very closely related and morphologically similar. The increase in the production of male progeny by Gelis mothers over time suggests a depletion in sperm number or viability with age. This is especially interesting, given that Gelis species are among the least fecund parasitoids thus far studied. It is likely that in the field most Gelis mothers are probably only able to parasitize a few hosts and to maintain the production of female offspring.

Keywords Realized lifetime fecundity - Longevity · Gelis $\cdot$ Hymenoptera $\cdot$ Secondary parasitoids

\section{Introduction}

Phylogeny and phylogenetic constraints are central to our understanding of ecology and evolutionary biology (McKitrick 1993). Phylogeny describes the evolutionary history and ecophysiological relationships among 
groups of organisms (Gould, 1977; Nei and Kumar 2000). In nature, closely related species generally exhibit traits that very strongly reflect their phylogeny, such as morphology, reproduction and development (Cheverud et al. 1985; Blomberg et al. 2003). Consequently, closely related species generally exhibit a suite of traits that overlap and which are functionally specialized (West-Eberhard, 1986). Phylogenetic constraints are defined as components or aspects of a lineage that prevent or impede any anticipated form of evolution in that lineage (McKitrick 1993). In other words, a constraint affects the potential of a species to evolve radical changes in its biology and/or ecology from its ancestors and close relatives and may hinder a species attempting to exploit novel resources or colonize novel habitats. For instance, in many families of the Hymenoptera (Aculeata) the ovipositor, which is a specialized egglaying apparatus, has evolved into a stinger that instead of eggs injects venom into potential prey or as a means of defense against attackers (Zhao et al. 2015). A stinger is of much more utility than an ovipositor for a social wasp, because the workers are sterile and do not reproduce. In vertebrates, woodpeckers possess sharp, pointed bills for chiseling wood for nest construction and foraging, and long, barbed tongues made up of cartilage and bone for catching insects embedded in trees (Bock 1999; Zhou et al. 2009). These adaptations are specifically tailored for nesting in forest habitats and would clearly be of little utility in grasslands.

Phylogeny is not the only factor constraining organisms in nature. All species are also constrained in their ability to allocate limited metabolic resources for vital functions such as reproduction, foraging and survival (Roff 2002). Under strong selection for the acquisition, utilization and allocation of these resources to different and potentially competing fitness functions, the optimal phenotype of most organisms is determined by tradeoffs in life-history traits such as between reproduction and longevity (van Noordwijk and de Jong 1986). A trade-off occurs when two fitness-related traits are limited by the same resource, such as time and/or energy, that can only be utilized once (Stearns 1989). For example, an organism that invests a disproportionate amount of metabolic resources towards early reproduction will invariably experience a reduction in its life expectancy and vice-versa. Trade-offs strongly reflect environmental limitations on food, prey, mates and other factors. Thus, a specialized herbivore that feeds on a rare or small species of plant may be limited in its reproductive capacity by a difficulty in finding many food plants and therefore invests more metabolic resources in maintenance than in egg production, allowing it to extend its lifespan in searching for suitable plants on which to oviposit.

Parasitic wasps, or 'parasitoids', are model organisms for addressing a range of questions in evolutionary biology. Parasitoids lay their eggs inside or on the bodies of other arthropods ('hosts') and their larvae grow and develop by feeding on host tissues, whereas the adult stage is free-living (Godfray 1994). The development of parasitoid offspring is dependent on resources contained in a single host: because hosts of many parasitoids are only marginally bigger than the parasitoid itself, they are under intense selection to optimize the exploitation of limited resources to vital metabolic functions (Harvey 2005; Jervis et al. 2008). By contrast, selection for resource allocation to different metabolic functions in arthropod predators is generally much more relaxed, because they are not restricted to a single prey item. For this reason, parasitoids exhibit a suite of traits that are adapted for exploiting a narrow range of host species in nature, whereas most predators will attack many prey species in order to reach maturity.

Parasitoids that are closely related and/or exhibit similar host ranges in nature often exhibit a convergence in traits such as host utilization and reproduction (Jervis et al. 2008). This is because of both phylogenetic conservatism and overlapping selection pressures on tradeoffs such as between maintenance and egg production. One of the best examples on how host ecology drives similarities and differences in parasitoid traits is a study by Price (1972), who compared parasitoids attacking different stages of the Jack pine sawfly, Neodiprion swaineii. He found that parasitoids attacking early host stages, such as young larvae, exhibited traits such as the production of large numbers of small eggs that could be oviposited rapidly and adults that had short lifespans. By contrast, parasitoids attacking older hosts, such as pupae, produced small numbers of large eggs that took a considerable time to lay and adults that had extended lifespans. He attributed these differences to variation in mortality risks experienced by hosts along their ontogenetic continuum with high fecundity being an adaptive response in parasitoids attacking comparatively abundant young hosts with high mortality risks, and lower fecundity for parasitoids attacking scarce older hosts with a lower mortality risks (Price 1972; see also Pexton and Mayhew 2002). Differences in reproductive 
investment and longevity also appear to reflect host abundance (Price 1972).

Sex determination in parasitoids is haplodiploid, where unfertilized (haploid) eggs develop into males and fertilized (diploid) eggs develop into females. This gives females the choice of deciding what sex to lay during oviposition. In parasitoids, numerous studies have shown that mothers prefer to oviposit daughters on larger hosts of perceived higher quality, and sons on smaller hosts (Charnov et al. 1981; Charnov 1982; King 1987, 1989). This is because eggs are typically more costly to produce than sperm, with large females gaining much higher fitness returns than large males (Jervis et al. 2008). Importantly, offspring sex ratios in parasitoids in the field are dependent on more than host quality, such as population-related factors. For example, when plenty of resources are available, mating patterns in parasitoid populations generally select for equal parental investment into both sexes. However, when vital resources, such as mates or hosts, are scarce or limiting, increased competition can generate conditions that bias sex ratios in the direction of either sex (King 1987; Visser et al., 2016).

In this study we examined development and reproductive strategies in three congeneric species of parasitoids. Gelis agilis Fabricius, G. proximus Forster and G. hortensis Christ (Hymenoptera: Ichmeumonidae) are three species of facultative hyperparasitoids that are virtually morphologically and behaviorally indistinguishable, apart from differences in cuticle color. The three species exhibit a suite of overlapping traits that are phylogenetically conserved. These include (1) the production of large, yolky anhydropic eggs that are only produced in small numbers daily (Jervis \& Kidd 1986). (2) Obligate host-feeding behavior among adult females to secure proteins for oogenesis (Jervis and Kidd, 1986; Heimpel and Collier 1996). (3) Complete synovigeny where adult females emerge with no mature eggs (Jervis et al. 2001). (4) Ectoparasitic idiobisis, whereby eggs are laid by female parasitoids on the body surface of paralyzed hosts (Mayhew and Blackburn, 1999). (5) Wingless adults, which contrasts with most parasitoid taxa that are fully winged (Schwarz and Shaw 1999; Harvey et al. 2018). Despite these overlapping traits, the three species successfully co-occur in grassy meadow and forest edges across much of Eurasia, including the Netherlands. Little is known about the ecology or host range of most gelines, although previous work has shown that the three species studied here attack cocoons of primary parasitoids (Cotesia spp.) in the field (Lei and Hanski 1997; van Nouhuys and Hanski 2000; Harvey et al. 2014; Heinen and Harvey 2019). Thus far 280 species of Gelis have been described (Catalogue of Life, 2019), although there are certainly many more as the genus is not well-studied.

We compared fecundity schedules, lifetime reproductive success and offspring sex ratios in the three Gelis species when reared on cocoons of the gregarious parasitoid, Cotesia glomerata L. (Hyemoptera: Braconidae). Previous work with different Gelis species, including the wingless $G$. acororum and the fully winged $G$. areator, reported significant interspecific differences in fecundity schedules over two day periods and lifetime reproductive success, especially between the winged and wingless gelines. Furthermore, sex ratios in both species were highly male-biased throughout reproductive life (Visser et al. 2014, 2016). The authors speculated that the male bias was attributable to anticipation of limited hosts in nature by both parasitoids, with a high production of males reducing competition for these scarce hosts among the low number of remaining females (Visser et al. 2014). Given that the three species studied here are all wingless, we hypothesize that reproduction and longevity among them will be more similar than among the gelines in the Visser et al. (2014, 2016) studies. We also discuss how these several closely-related species exhibiting overlapping traits are able to co-exist locally at small scale in nature.

\section{Methods and Materials}

Insects

All insects were reared at a temperature of $22 \pm 2{ }^{\circ} \mathrm{C}$ under a 16:8 h L:D regime with a relative humidity of $50 \%$. Cultures of the parasitoid C. glomerata and its host, the large cabbage white butterfly $P$. brassicae, were obtained from insects reared at Wageningen University (WUR), the Netherlands, that were originally collected from agricultural fields in the vicinity of the University. All C. glomerata cocoons used in this experiment were generated from $P$. brassicae caterpillars reared on Brassica oleracea var. Cyrus (Brussels sprouts) at the Netherlands Institute of Ecology (NIOO, Wageningen, The Netherlands).

In the field, C. glomerata females generally oviposit between 10 and 40 eggs into first (L1) to third (L3) 
instars of $P$. brassicae. Fully grown parasitoid larvae emerge from the host caterpillar late during its final instar, spinning cocoons adjacent to the host, which perishes within a few days. Once weekly, several hundred L2 P. brassicae larvae were presented to C. glomerata in rearing cages $(30 \times 30 \times 30 \mathrm{~cm})$ for parasitism. Parasitized caterpillars were then transferred to steel and plexiglass cages $(30 \times 30 \times 60 \mathrm{~cm})$ containing cabbage plants. Fresh parasitoid cocoons were collected from these cages.

Adult G. agilis, G. proximus and G. hortensis were collected from cocoons of $C$. glomerata that had been pinned onto the lower shoots and stems of black mustard (Brassica nigra) or garlic mustard (Alliaria petiolata) at several locations in the provinces of Gerlderland and South Holland, the Netherlands. Species identification was made by Dr. Martin Schwarz, one of the world's leading experts on the Cryptinae, who is based at the Biologiezentrum in Linz, Austria. A recent phylogenetic reconstruction of the three geline species is shown in Harvey et al. (2018).

Each species was therefore obtained from multiple populations that were reared collectively. In culture, hyperparasitoids were maintained exclusively on fresh cocoons of $C$. glomerata. After adult emergence, each species was separately kept in closed, meshed rearing cages $(30 \times 30 \times 30 \mathrm{~cm})$ with honey and water and stored at $10 \pm 1{ }^{\circ} \mathrm{C}$ in incubators.

Fecundity Schedules, Lifetime Reproductive Success, Longevity and Sex Allocation

Newly emerged females of G. proximus and $G$. hortensis were sexed, fed with honey and allowed to mate with males in Petri dishes $(8 \mathrm{~cm}$ dia.). After mating, which was ascertained visually, females were transferred to new Petri dishes (12 cm dia.) containing 10 cocoons of $<12$ h-old $C$. glomerata with drops of honey smeared on the underside of the lid and water absorbed into a small ball of cotton wool. Every $48 \mathrm{~h}$ cocoons were removed and placed in marked vials with the species, female number, dates, and days of exposure marked on the vials. Fresh cocoons, honey and water were presented to female wasps in new Petri dishes. This procedure was repeated throughout the lives of female wasps. Vials were checked daily for adult eclosion and newly emerged wasps were sexed and counted. These data make it possible to determine fecundity schedules of the three wasps, total fecundity, offspring sex ratios over time and longevity. The experiment was repeated with 10 females of each species. In order to measure longevity for female wasps without host access (=control), the procedure was repeated with 10 females of each species in Petri dishes without host access. To measure male longevity, the procedure was repeated in Petri dishes with 10 males each of the sexual species G. proximus and G. hortensis.

\section{Statistics}

All statistics were performed using IBM SPSS Statistics (IBM Corp. Released 2017. IBM SPSS Statistics for Windows, Version 25.0. Armonk, NY: IBM Corp). To compare total number of offspring and longevity of females provided with hosts, we used general linear model ANOVAs with species as main factor. Offspring numbers were log-transformed to meet assumptions of equal variance. If the effect of species was significant, means were compared using Tukey multiple comparison tests (Fig. 1).

\section{Results}

Fecundity Schedules and Lifetime Reproductive Success in the Three Geline Wasps

Total offspring numbers differed among the three species $\left(\mathrm{F}_{2,26}=15.5, p<0.001\right)$, with $G$. proximus producing most offspring $(126.7 \pm 14.5$, mean $\pm 1 \mathrm{SE})$, followed by G. hortensis $(87.5 \pm 9.2)$ and G. agilis producing the fewest total offspring $(41.9 \pm 4.5)$ (Fig. 2). Total reproduction was also reflected in the fecundity schedules of the three species. Gelis agilis had a preoviposition period after eclosion of several days and then typically produced a maximum of $\sim 2$ progeny every two days until this declined around day 50. By contrast, G. hortensis had the shortest pre-ovipositon period of the three species and produced a maximum of $\sim 3-4$ progeny every two days which dropped off rapidly after around 35 days. Lastly, the pre-ovipositon period of $G$. proximus was intermediate in length and this species also produced $\sim 3-4$ progeny every two days, but the decline in progeny production was much more gradual than in $G$. hortensis, and also dropped off sharply only after around 90 days (Fig. 3). 

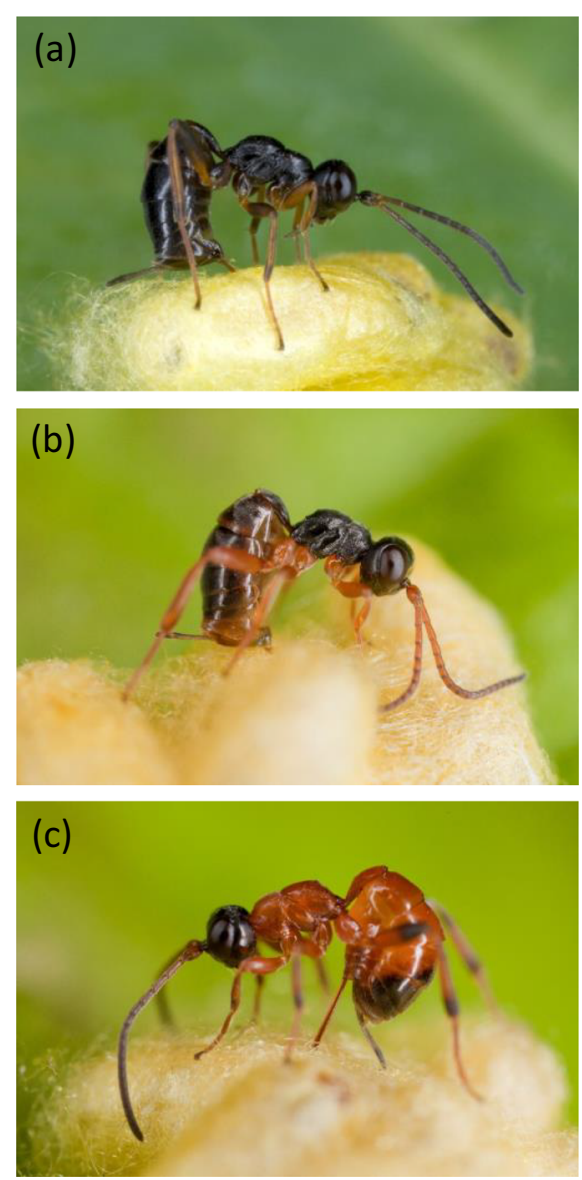

Fig. 1 Adult females of Gelis agilis (a), Gelis proximus (b) and Gelis hortensis (c) parasitizing cocoons of Cotesia glomerata

\section{Longevity}

Although G. agilils tended to have a shorter lifespan than the other two species, the longevity of females provided with hosts did not statistically differ among them $\left(\mathrm{F}_{2,26}=2.26, p=0.12\right.$, Fig. 4$)$. Each of the three species lived on average between 80 and 120 days. At the upper end of the longevity distribution, some G. proximus females lived for almost 6 months.

\section{Sex ratios}

Overall sex ratios did not differ between the two sexually reproducing species, $G$. proximus and $G$. hortensis $\left(\mathrm{F}_{1,18}=1.30, p=0.270\right)$, but allocation of male and female progeny did differ throughout the life of each species (Likelihood Ratio Test $=19.33, p<0.0001$; Fig. 5). In G. proximus there was a much more dramatic shift from the production of female to male offspring later in reproductive life than in $G$. hortensis.

\section{Discussion}

Females of the three Gelis species are morphologically similar, aside from differences in the color of their cuticle (Fig. 1). Despite these similarities, we found that there were interspecific differences in fecundity schedules and lifetime reproductive success among the three species. Total progeny production was significantly lower in G. agilis than in the other two Gelis species; female $G$. proximus produced three times as many progeny as $G$. agilis females, with $G$. hortensis producing intermediate numbers of progeny. Moreover, despite the fact that the three species only produce eggs in small numbers, a peak in mean number of progeny produced over two days was higher in $G$. proximus and G. hortensis $(\sim 3-4)$ than in G. agilis $(\sim 1-2)$. However, progeny production declined in $G$. hortensis somewhat more rapidly than in G. proximus. Longevity among females provided with hosts did not differ significantly among the three gelines.

Our study shows that there are both interspecific similarities and differences among the three gelines in terms of trait expression. Traits like behavior, general appearance (morphology, winglessness) and physiology (ectoparasitism, host-feeding and the production of small numbers of large, yolky eggs) were present in all three species and are typical among most species in the Gelinae (Schwarz and Shaw 1999). However,

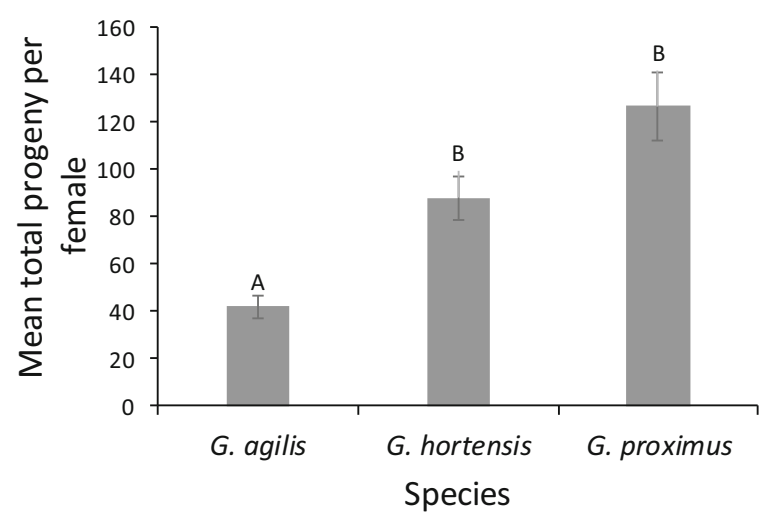

Fig. 2 Mean total progeny ( \pm SE) produced by females of Gelis agilis, Gelis proximus, and Gelis hortensis. Data from 10 adult females for G. proximus and G. hortensis and 9 adult females for G. agilis. Bars with different letters are statistically different (Tukey tests, $p<0.05$ ) 
Fig. 3 Fecundity schedules ( \pm SE) of (a) Gelis agilis (b) Gelis hortensis (c) Gelis proximus. Data from 10 adult females for G. proximus and G. hortensis and 9 adult females for $G$. agilis a)

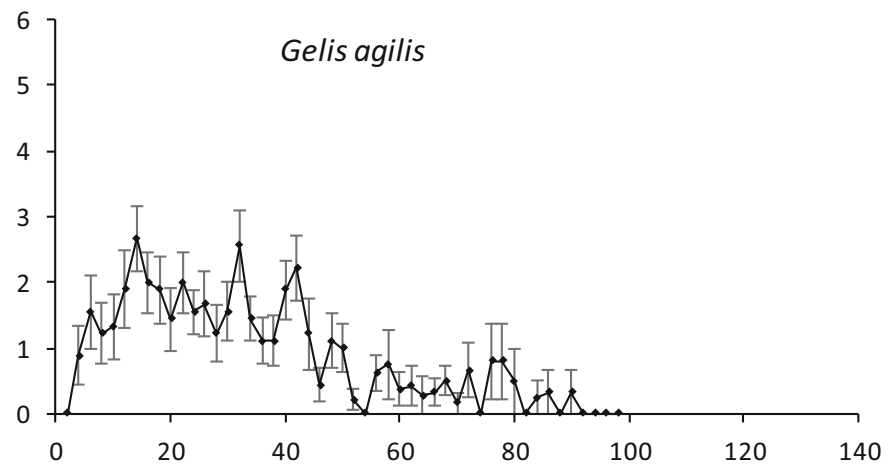

b)
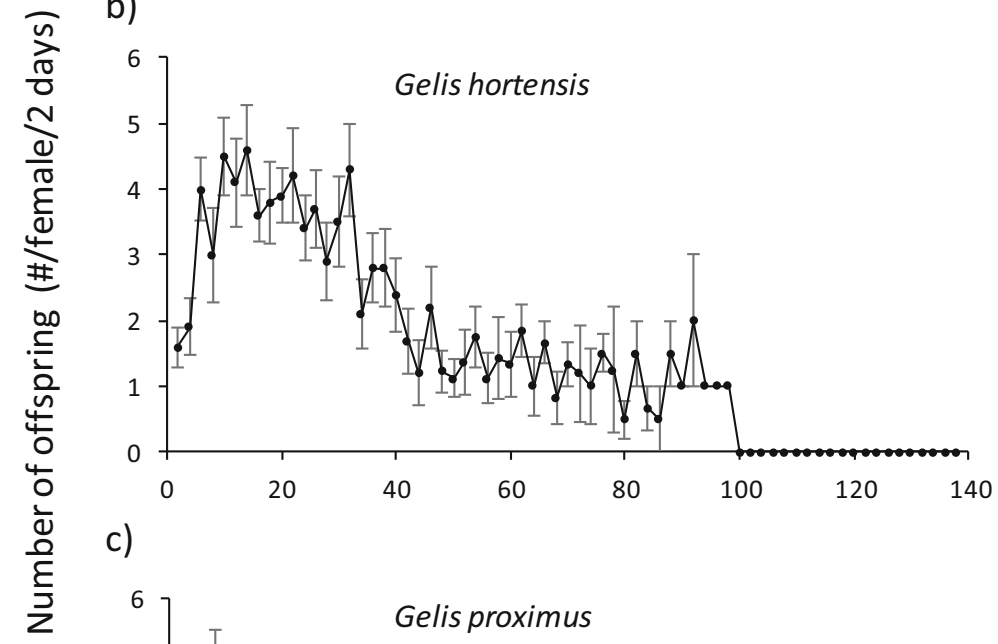

c)

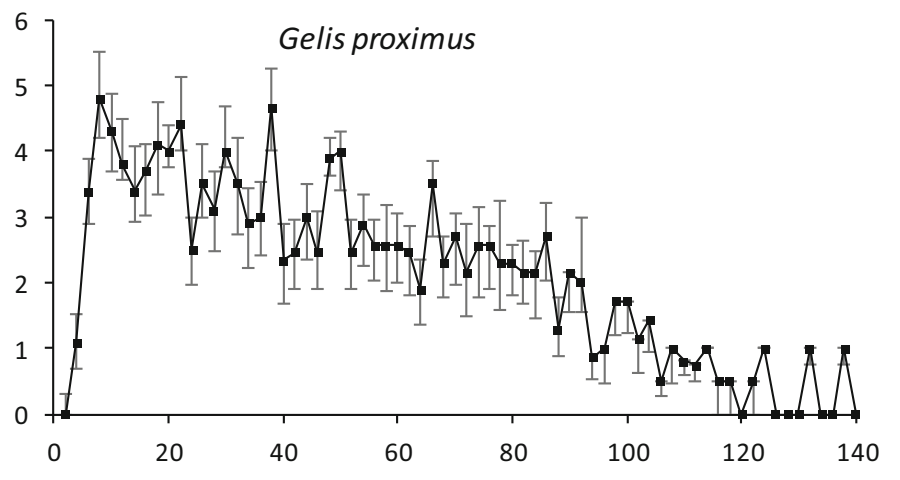

Female age (days post emergence) differences in longevity and reproduction, although subtle when compared with many more distantly related parasitoids (e.g. koinobionts) were nevertheless observed. This suggests that selection on these traits differs among the three species. It is difficult to understand what these divergent selection pressures might be, although trade-offs may be involved. For instance, Gelis species may differ in their competitive (intrinsic and extrinsic) abilities, with the better competitor producing less offspring (Harvey et al. 2013). On the other hand, differences in reproduction may simply reflect marginal differences in host range or else some aspect of niche differentiation. Gelis spp. are generalists and can probably attack a range of hosts in nature (in addition to the cocoons of other parasitoids). However, the lack of wings in most gelines probably limits the number of 


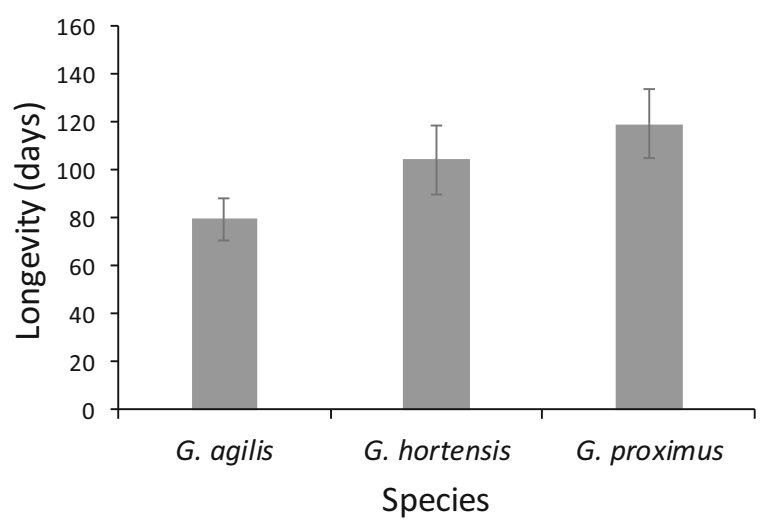

Fig. 4 Mean longevity ( \pm SE) of females of Gelis agilis, Gelis proximus, and Gelis hortensis when provided with hosts. Data from 10 adult females for $G$. proximus and G. hortensis and 9 adult females for G. agilis

suitable hosts they are able to find and parasitize in the field. In spite of this, in the.

Netherlands, the three species studied here co-occur in the same habitats with at least two other morphologically similar species, G. acororum and G. spurius, and perhaps more (Harvey et al. 2014).

Offspring sex ratios in G. proximus and G. hortensis were highly variable among individual females, at least during the first several weeks of reproductive life. In most $G$. hortensis females, offspring sex ratios favored the production of sons over this time $(>70 \%)$, whereas in G. proximus ca. $60 \%$ of progeny were daughters. However, in both species there was an abrupt shift from the production of mixed sex to all-male offspring after about 40 days, leading to the production of all-male progeny later in life. There are two possible explanations. The first, and less likely, is that sperm is unlimited, but that females produce more daughters early to gain a competitive edge for access to potentially limiting hosts in the field. This argument assumes, however, that adult cohorts of different Gelis species strongly overlap temporally and that host availability decreases rapidly over time. Consequently, the first daughters that emerge have the greatest chance of exploiting hosts before they are depleted. This scenario is highly unlikely because gelines are long-lived and there are probably no discrete generations in nature but a constant turnover of wasps as long as hosts are available. In another laboratory experiment, Visser et al. (2014) reported male-biased sex ratios throughout life in two other Gelis species, the wingless $G$. acororum and the winged $G$. areator. The authors attributed the male bias in these species to a perceived scarcity of hosts in the field, meaning that just enough daughters are produced to diffuse competition among the parasitoids.

The second and more likely explanation is that G. proximus and G. hortensis are sperm-limited and thus deplete their sperm stores abruptly. For many years it was assumed that eggs are much more limiting than sperm in parasitoids, and this notion was often used as the basis of optimization models for sex allocation strategies in these insects (Charnov 1982; King 1987, 1989; Visser 1994; Mackauer, 1996). However, it has more recently been revealed that many female parasitoids mate only once in their lifetimes and store low numbers of sperm (Henter 2004; Boivin et al. 2005; Boivin 2013). If males inseminate females with just enough sperm to produce daughters early in reproductive life, this may imply that Gelis spp. will only ever encounter small numbers of hosts and that the sudden switch to allmale progeny is an artefact: in nature, no females therefore survive long enough to encounter enough hosts to
Fig. 5 Sex allocation patterns (proportion of males, mean $\pm \mathrm{SE}$ ) during life of Gelis hortensis (blue line) and Gelis proximus (orange line). Sex ratios were determined for progeny produced by 10 females of each species in consecutive 6-day intervals

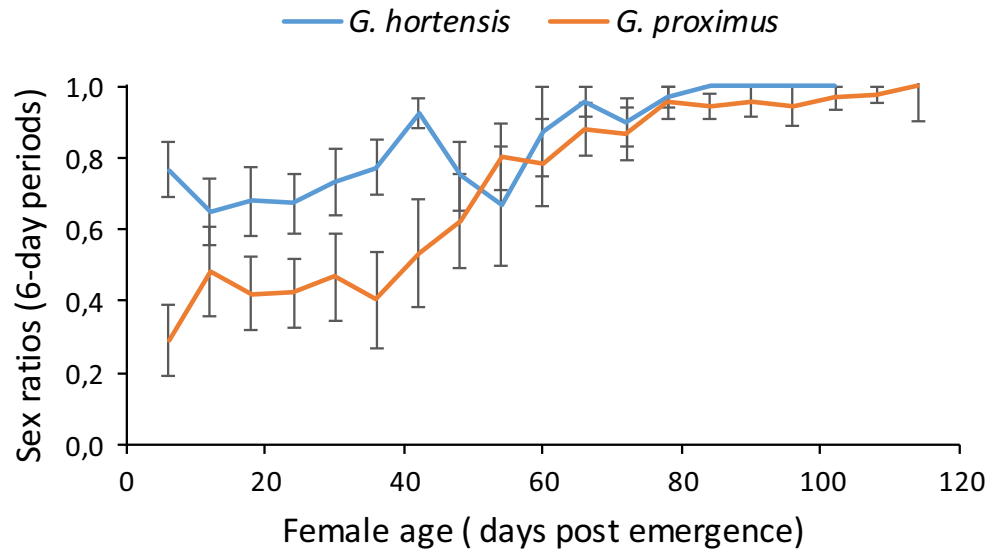


deplete their sperm. What is remarkable in G. proximus and $G$. hortensis is that the switch from mixed broods to all-male progeny occurred when females had only laid around 50 or so eggs - much lower than in other parasitoids so far studied. However, given that suitable hosts are probably scarce it is unlikely that females ever exhaust their supply of sperm in nature. Our experimental females thus experienced a 'jackpot' of hosts that far exceeds what females would normally ever encounter. Males thus inseminate just enough sperm to ensure that females do not become sperm-depleted.

Given that at least several Gelis species are found in the same microhabitats, an interesting question is how multiple species that presumably compete for hosts at small, local scales can co-exist. One important assumption in ecology is that co-existing species differ in their niches (Adler et al., 2007) and that intense competition among similar species for the same resources, such as hosts by parasitoids, will lead to competitive exclusion of others by a dominant species (Harvey et al. 2013; Pekas et al. 2016). Co-existence theory has been posited as a framework to better understand how species with strongly overlapping traits can co-exist in ecologically similar environments (Leibold and McPeek, 2006). This theory may partly explain niche differentiation among species with overlapping traits. One important factor that may enable multiple Gelis species to co-exist locally is that they are extreme generalists, parasitizing whatever suitable hosts are available. Although the biology of most Gelis species is unknown, other species in this genus parasitize a wide range of hosts including spider egg sacs and moth pupae in addition to parasitoid cocoons (Bezant 1956; Schwarz \& Boriani 1994; Cobb and Cobb 2004). They may also differ in other, more subtle ways that diffuse competition. For instance, species may co-exist by differing their usage of limiting resources, their ability to colonize habitats and their temporal patterns of habitat occupancy (Leibold and McPeek 2006; Adler et al. 2007). Furthermore, interspecific differences in egg production, sex allocation and fecundity may reflect subtle differences in host preference or host-finding ability that will be the focus of future research in the field.

Acknowledgements The authors with to thank Arjen de Jong for help in collecting gelines in the field. Photos of the gelines in Fig. 1 are by Tibor Bukovinszky. Roel Wagenaar is thanked for the rearing of Cotesia glomerata and the three Gelis species.
Data Accessibility Upon acceptance of the manuscript all data will be archived in the Dryad Respository.

\section{Compliance with ethical standards}

Conflict of interests The authors declare that they have no conflicts of interest.

Open Access This article is distributed under the terms of the Creative Commons Attribution 4.0 International License (http:// creativecommons.org/licenses/by/4.0/), which permits unrestricted use, distribution, and reproduction in any medium, provided you give appropriate credit to the original author(s) and the source, provide a link to the Creative Commons license, and indicate if changes were made.

\section{References}

Adler PB, HilleRisLambers J, Levine JM (2007) A niche for neutrality. Ecol Lett 10:95-104

Bezant ET (1956) Gelis micrurus Forster (Hymenoptera: Ichneumonidae) parasitising a lycosid spider egg sac. Entomol Month Mag 92:106

Blomberg SP, Garland T, Ives AR (2003) Testing for phylogenetic signal in comparative data: behavioral traits are more labile. Evolution 57:717-745

Bock WJ (1999) Functional and evolutionary morphology of woodpeckers. Ostrich 70:23-31

Boivin G, Jacob S, Damiens D (2005) Spermatogeny as a lifehistory index in parasitoid wasps. Oecologia 143:198-202

Boivin G (2013) Sperm as a limiting factor in mating success in Hymenoptera parasitoids. Entomol Exp et Appl 146:149155

Catalogue of Life (2019) 2019 Annual Checklist. Gelis http://www.catalogueoflife.org/col/browse/tree/id/7555800 b1216a3f5e8e4e740288c8463

Charnov EL, Los-den Hartogh RL, Jones WT, van den Assem J (1981) Sex ratio evolution in a variable environment. Nature 289:27-33

Charnov EL (1982) The Theory of Sex Allocation. Volume 18. Princeton University Press

Cheverud JM, Dow MM, Leutenegger W (1985) The quantitative assessment of phylogenetic constraints in comparative analyses: sexual dimorphism in body weight among primates. Evolution 39:1335-1351

Cobb LM, Cobb VA (2004) Occurrence of parasitoid wasps, Baeus sp. and Gelis sp., in the egg sacs of the wolf spiders Pardosa moesta and Pardosa sternalis (Araneae, Lycosidae) in southeastern Idaho. Canadian Field Naturalist 118:122123

Godfray HCJ (1994) Parasitoids: Behavioral and Evolutionary Ecology. Princeton University Press

Gould SJ (1977) Ontogeny and Phylogeny. Harvard University Press

Harvey JA (2005) Factors affecting the evolution of development strategies in parasitoid wasps: the importance of functional constraints and incorporating complexity. Entomol Exp Appl 117:1-13 
Harvey JA, Poelman EH, Tanaka T (2013) Intrinsic inter-and intraspecific competition in parasitoid wasps. Annu Rev Entomol 58:333-351

Harvey JA, Snaas H, Malcicka M, Visser B, Bezemer TM (2014) Small-scale spatial resource partitioning in a hyperparasitoid community. Arthropod Plant Interact 8:393-401

Harvey JA, Visser B, Lammers M, Marien J, Gershenzon J, Ode PJ, Heinen R, Gols R, Ellers J (2018) Ant like traits in wingless parasitoids repel attack from wolf spiders. J Chem Ecol 44:894-904

Heimpel GE, Collier TR (1996) The evolution of host-feeding behaviour in insect parasitoids. Biol Rev 71:373-400

Heinen R, Harvey JA (2019) Spatial and temporal diversity in hyperparasitoid communities of Cotesia glomerata on garlic mustard, Alliaria petiolata. Ecol Entomol 44:357-366

Henter HJ (2004) Constrained sex allocation in a parasitoid due to variation in male quality. J Evol Biol 17:886-896

Jervis MA, Kidd NAC (1986) Host-feeding strategies in hymenopteran parasitoids. Biol Rev 61:395-434

Jervis MA, Heimpel GE, Ferns PN, Harvey JA, Kidd NAC (2001) Life-history strategies in parasitoid wasps: a comparative analysis of 'ovigeny'. J Anim Ecol 70:442-458

Jervis MA, Ellers J, Harvey JA (2008) Resource acquisition, allocation, and utilization in parasitoid reproductive strategies. Annu Rev Entomol 53:361-385

King BH (1987) Offspring sex ratios in parasitoid wasps. Q Rev Biol 62:367-396

King BH (1989) Host-size-dependent sex ratios among parasitoid wasps: does host growth matter? Oecologia 78:420-426

Leibold MA, McPeek MA (2006) Coexistence of the niche and neutral perspectives in community ecology. Ecology 87 : $1399-1410$

Lei GC, Hanski I (1997) Metapopulation structure of Cotesia melitaearum, a specialist parasitoid of the butterfly Melitaea cinxia. Oikos 78:91-100

Mackauer M (1996) Sexual size dimorphism in solitary parasitoid wasps: influence of host quality. Oikos 76:265-272

Mayhew PJ, Blackburn TM (1999) Does development mode organize life-history traits in the parasitoid Hymenoptera? J Anim Ecol 68:906-916

McKitrick MC (1993) Phylogenetic constraint in evolutionary theory: has it any explanatory power? Ann Rev Ecol System 24:307-330

Nei M, Kumar S (2000) Molecular Evolution and Phylogenetics. Oxford University Press

Pekas A, Tena A, Harvey JA, Garcia-Marí F, Frago E (2016) Host size and spatiotemporal patterns patterns mediate the coexistence of specialist parasitoids. Ecology 97:1345-1356
Pexton JJ, Mayhew PJ (2002) Siblicide and life-history evolution in parasitoids. Behav Ecol 13:690-695

Price PW (1972) Parasitiods utilizing the same host: adaptive nature of differences in size and form. Ecology 53:190-195

Roff DA (2002) Life History Evolution. Sinauer Associates, Sunderland

Schwarz M, Boriani M (1994) Redescription of Gelis longulus (Hymenoptera: Ichneumonidae), a parasitoid of Ocnerostoma piniariellum (Lepidoptera: Yponomeutidae). Eur J Entomol 91:331-331

Schwarz M, Shaw MR (1999) Western Palaeartic Cryptinae (Hymenoptera: Ichneumonidae) in the National Museums of Scotland, with nomenclatural changes, taxonomic notes, rearing records and special reference to the British check list. Part 2. Genus Gelis Thunberg (Phygadeuontini: Gelina). Entomol Gaz 50:117-142

Stearns SC (1989) Trade-offs in life-history evolution. Funct Ecol 3:259-268

van Noordwijk AJ, de Jong G (1986) Acquisition and allocation of resources: their influence on variation in life history tactics. Am Nat 128:137-142

van Nouhuys S, Hanski I (2000) Apparent competition between parasitoids mediated by a shared hyperparasitoid. Ecol Lett 3: $82-84$

Visser B, Le Lann C, Snaas H, Hardy ICW, Harvey JA (2014) Consequences of resource competition for sex allocation and discriminative behaviors in a hyperparasitoid wasp. Behav Ecol Sociobiol 68:105-113

Visser B, Le Lann C, Snaas H, Verdeny-Vilalta O, Harvey JA (2016) Divergent life history strategies in congeneric hyperparasitoids. Evol Ecol 30:535-549

Visser ME (1994) The importance of being large: the relationship between size and fitness in females of the parasitoid Aphaereta minuta (Hymenoptera: Braconidae). J Anim Ecol 63:963-978

West-Eberhard M (1986) Alternative adaptations, speciation, and phylogeny (a review). Proc Natl Acad Sci 83:1388-1392

Zhao ZL, Zhao HP, Ma GJ, Wu CW, Yang K, Feng XQ (2015) Structures, properties, and functions of the stings of honey bees and paper wasps: a comparative study. Entomol Month Mag 4:921-928

Zhou P, Kong XQ, Wu CW, Chen Z (2009) The novel mechanical property of tongue of a woodpecker. J Bionic Eng 6:214-218

Publisher's note Springer Nature remains neutral with regard to jurisdictional claims in published maps and institutional affiliations. 